\title{
A distributional study of negated adjectives and antonyms
}

\section{Supplementary materials}

\author{
Laura Aina, Raffaella Bernardi, Raquel Fernández
}

\section{Annotation of lexical antonyms: contraries vs. contradictory}

Data Each annotator is given a list of 148 adjectives pairs with opposite meanings (antonyms). The list is obtained according to the following criteria:

- The adjectives are tagged as regular antonyms in the Lexical Negation dictionary (van Son, van Miltenburg);

- Both the adjectives occur more than 100 times in the training corpus of the distributional model (UkWac + Wacky);

- The negated adjective of at least one of the members of the pair occurs more than 100 times in the training corpus of the distributional model (UkWac + Wacky).

Annotation guidelines Given a pair of words $a_{1}$ and $a_{2}$, the annotator considers the following sentence pattern:

(a) $X$ is neither $a_{1}$ nor $a_{2}$

- The annotator tags the pair as $\mathrm{x}$ if she does not know the meaning of the word, or does not interpret the two words as having opposite meanings.

- The annotator tags the pair as 1 (contrary) if (a) is acceptable under a default context.

- The annotator tags the pair as 0 (contradictory) if (a) is not acceptable under a default context.

The annotator is asked to carry out the task following for each case her first intuitive judgement.

Agreement Interraters agreement is computed using Fleiss' $k$, which generalizes Cohen's $k$ to cases with more than two coders.

- Agreement on the three categories (1, $0, \mathrm{x})$ : Fleiss' $k=0.38$;

- Agreement on two categories $(1,0)$ : : Fleiss' $k=0.37$.

Dataset For the purpose of the final categorization used in the analyses, only pairs that have been categorized as either 1 or 0 are considered, hence discarding those for which at least one annotator tagged them as $\mathrm{x}$, or for which there was not full agreement.

\section{Examples of adjective pairs and their annotation}

$\begin{array}{ll}\text { beautiful, ugly } & \text { contraries } \\ \text { alive, dead } & \text { contradictory } \\ \text { free, bound } & \text { neither } \\ \text { actual, potential } & \text { neither }\end{array}$




\section{Antonyms and negated adjective triples}

The list of triples is also provided as a csv file at: https://lauraina.github.io/data/notadj.csv

\subsection{Lexical antonyms}

Triple

Class

Frequency of negated adjective

\begin{tabular}{|c|c|c|c|c|}
\hline negligent & diligent & not negligent & - & 104 \\
\hline fair & foul & not fair & - & 1597 \\
\hline present & absent & not present & contradictory & 6621 \\
\hline present & absent & not absent & contradictory & 112 \\
\hline hot & cold & not hot & contraries & 305 \\
\hline hot & cold & not cold & contraries & 265 \\
\hline active & extinct & not active & - & 773 \\
\hline warm & cool & not warm & contraries & 106 \\
\hline warm & cool & not cool & contraries & 217 \\
\hline smooth & rough & not smooth & - & 163 \\
\hline sharp & flat & not sharp & contraries & 142 \\
\hline sharp & flat & not flat & contraries & 225 \\
\hline natural & flat & not natural & - & 590 \\
\hline natural & flat & not flat & - & 225 \\
\hline straight & crooked & not straight & - & 222 \\
\hline hard & soft & not hard & - & 2539 \\
\hline hard & soft & not soft & - & 120 \\
\hline long & short & not long & contraries & 4211 \\
\hline long & short & not short & contraries & 664 \\
\hline close & distant & not close & - & 516 \\
\hline national & international & not national & - & 207 \\
\hline national & international & not international & - & 145 \\
\hline prescriptive & descriptive & not prescriptive & - & 217 \\
\hline fast & slow & not fast & - & 123 \\
\hline fast & slow & not slow & - & 461 \\
\hline full & empty & not full & contraries & 943 \\
\hline full & empty & not empty & contraries & 419 \\
\hline military & civilian & not military & - & 177 \\
\hline civil & sidereal & not civil & - & 174 \\
\hline loud & soft & not loud & contraries & 101 \\
\hline loud & soft & not soft & contraries & 120 \\
\hline uniform & multiform & not uniform & contradictory & 499 \\
\hline present & past & not present & contraries & 6621 \\
\hline present & future & not present & contraries & 6621 \\
\hline sharp & dull & not sharp & - & 142 \\
\hline early & late & not early & contraries & 268 \\
\hline early & late & not late & contraries & 321 \\
\hline hungry & thirsty & not hungry & - & 196 \\
\hline linear & planar & not linear & - & 250 \\
\hline offensive & defensive & not offensive & contraries & 209 \\
\hline active & passive & not active & - & 773 \\
\hline active & passive & not passive & - & 217 \\
\hline simple & compound & not simple & contradictory & 625 \\
\hline single & multiple & not single & contradictory & 280 \\
\hline linear & cubic & not linear & - & 250 \\
\hline functional & organic & not functional & - & 121 \\
\hline dominant & recessive & not dominant & - & 107 \\
\hline
\end{tabular}




\begin{tabular}{|c|c|c|c|c|}
\hline foreign & native & not foreign & contradictory & 153 \\
\hline foreign & native & not native & contradictory & 664 \\
\hline extensive & intensive & not extensive & - & 200 \\
\hline plain & fancy & not plain & contraries & 119 \\
\hline plain & fancy & not fancy & contraries & 236 \\
\hline top & side & not top & contraries & 252 \\
\hline common & individual & not common & - & 1858 \\
\hline common & individual & not individual & - & 497 \\
\hline wise & foolish & not wise & contraries & 373 \\
\hline soft & hardened & not soft & contraries & 120 \\
\hline flat & contrasty & not flat & - & 225 \\
\hline found & lost & not found & - & 366 \\
\hline exclusive & inclusive & not exclusive & - & 1089 \\
\hline hostile & amicable & not hostile & contraries & 162 \\
\hline ripe & green & not ripe & contraries & 127 \\
\hline ripe & green & not green & contraries & 154 \\
\hline local & general & not local & - & 500 \\
\hline local & general & not general & - & 216 \\
\hline primary & secondary & not primary & - & 123 \\
\hline new & worn & not new & - & 2965 \\
\hline safe & dangerous & not safe & - & 1741 \\
\hline safe & dangerous & not dangerous & - & 521 \\
\hline robust & frail & not robust & - & 239 \\
\hline right & center & not right & contraries & 1620 \\
\hline compliant & defiant & not compliant & - & 173 \\
\hline first & last & not first & contraries & 380 \\
\hline first & last & not last & contraries & 2668 \\
\hline heavy & light & not heavy & - & 255 \\
\hline heavy & light & not light & - & 206 \\
\hline central & peripheral & not central & contraries & 340 \\
\hline sad & glad & not sad & contraries & 147 \\
\hline early & middle & not early & - & 268 \\
\hline homogeneous & heterogeneous & not homogeneous & - & 125 \\
\hline actual & potential & not actual & - & 528 \\
\hline optional & obligatory & not optional & contradictory & 162 \\
\hline optional & obligatory & not obligatory & contradictory & 417 \\
\hline full & thin & not full & contraries & 943 \\
\hline old & young & not old & - & 818 \\
\hline old & young & not young & - & 209 \\
\hline physical & mental & not physical & - & 353 \\
\hline simple & complex & not simple & - & 625 \\
\hline simple & complex & not complex & - & 137 \\
\hline thick & thin & not thick & - & 109 \\
\hline live & recorded & not live & contradictory & 214 \\
\hline positive & neutral & not positive & contraries & 232 \\
\hline positive & neutral & not neutral & contraries & 250 \\
\hline attractive & repulsive & not attractive & contraries & 223 \\
\hline major & minor & not major & - & 229 \\
\hline direct & retrograde & not direct & - & 397 \\
\hline parallel & perpendicular & not parallel & contraries & 163 \\
\hline large & small & not large & contraries & 1764 \\
\hline large & small & not small & contraries & 486 \\
\hline most & least & not most & contraries & 996 \\
\hline most & least & not least & contraries & 15197 \\
\hline identical & fraternal & not identical & - & 1766 \\
\hline
\end{tabular}




\begin{tabular}{|c|c|c|c|c|}
\hline active & quiet & not active & contraries & 773 \\
\hline positive & negative & not positive & - & 232 \\
\hline positive & negative & not negative & - & 178 \\
\hline first & second & not first & contraries & 380 \\
\hline first & second & not second & contraries & 127 \\
\hline natural & sharp & not natural & - & 590 \\
\hline natural & sharp & not sharp & - & 142 \\
\hline solid & liquid & not solid & contraries & 159 \\
\hline free & bound & not free & - & 2270 \\
\hline absolute & relative & not absolute & - & 758 \\
\hline open & closed & not open & - & 3802 \\
\hline separate & joint & not separate & contradictory & 636 \\
\hline meaningful & meaningless & not meaningful & - & 154 \\
\hline proud & humble & not proud & contraries & 334 \\
\hline serious & frivolous & not serious & contraries & 854 \\
\hline wide & narrow & not wide & contraries & 266 \\
\hline religious & secular & not religious & contradictory & 568 \\
\hline genuine & counterfeit & not genuine & - & 383 \\
\hline strange & familiar & not strange & - & 251 \\
\hline strange & familiar & not familiar & - & 2970 \\
\hline native & adopted & not native & contradictory & 664 \\
\hline objective & subjective & not objective & contradictory & 144 \\
\hline natural & artificial & not natural & contradictory & 590 \\
\hline automatic & manual & not automatic & contradictory & 363 \\
\hline nuclear & conventional & not nuclear & - & 108 \\
\hline nuclear & conventional & not conventional & - & 131 \\
\hline strong & weak & not strong & - & 1860 \\
\hline strong & weak & not weak & - & 129 \\
\hline single & double & not single & - & 280 \\
\hline active & stative & not active & - & 773 \\
\hline square & round & not square & contraries & 173 \\
\hline external & internal & not external & - & 109 \\
\hline old & new & not old & contraries & 818 \\
\hline old & new & not new & contraries & 2965 \\
\hline more & less & not more & - & 10527 \\
\hline more & less & not less & - & 10235 \\
\hline real & nominal & not real & - & 1434 \\
\hline dominant & subordinate & not dominant & contraries & 107 \\
\hline tall & short & not tall & contraries & 142 \\
\hline tall & short & not short & contraries & 664 \\
\hline busy & idle & not busy & - & 333 \\
\hline busy & idle & not idle & - & 144 \\
\hline direct & inverse & not direct & - & 397 \\
\hline big & little & not big & contraries & 1349 \\
\hline big & little & not little & contraries & 136 \\
\hline brave & cowardly & not brave & contraries & 125 \\
\hline negative & neutral & not negative & contraries & 178 \\
\hline negative & neutral & not neutral & contraries & 250 \\
\hline active & dormant & not active & - & 773 \\
\hline foreign & domestic & not foreign & - & 153 \\
\hline solid & gaseous & not solid & - & 159 \\
\hline deep & shallow & not deep & - & 273 \\
\hline bright & dull & not bright & contraries & 169 \\
\hline private & public & not private & contradictory & 417 \\
\hline private & public & not public & contradictory & 533 \\
\hline
\end{tabular}




\begin{tabular}{|c|c|c|c|c|}
\hline true & false & not true & contradictory & 6524 \\
\hline true & false & not false & contradictory & 226 \\
\hline much & little & not much & contraries & 10706 \\
\hline much & little & not little & contraries & 136 \\
\hline abstract & concrete & not abstract & - & 144 \\
\hline rich & lean & not rich & - & 430 \\
\hline nice & nasty & not nice & contraries & 553 \\
\hline late & middle & not late & - & 321 \\
\hline live & dead & not live & contradictory & 214 \\
\hline live & dead & not dead & contradictory & 1244 \\
\hline beautiful & ugly & not beautiful & contraries & 200 \\
\hline wild & tame & not wild & - & 105 \\
\hline clear & opaque & not clear & - & 15096 \\
\hline tough & tender & not tough & - & 119 \\
\hline national & local & not national & contraries & 207 \\
\hline national & local & not local & contraries & 500 \\
\hline straight & curly & not straight & - & 222 \\
\hline blind & sighted & not blind & contradictory & 395 \\
\hline different & same & not different & - & 584 \\
\hline expensive & cheap & not expensive & - & 438 \\
\hline expensive & cheap & not cheap & - & 1238 \\
\hline innocent & guilty & not innocent & contradictory & 120 \\
\hline innocent & guilty & not guilty & contradictory & 4152 \\
\hline smart & stupid & not smart & contraries & 167 \\
\hline smart & stupid & not stupid & contraries & 439 \\
\hline fat & thin & not fat & contraries & 149 \\
\hline safe & out & not safe & - & 1741 \\
\hline difficult & easy & not difficult & - & 3480 \\
\hline difficult & easy & not easy & - & 9747 \\
\hline near & far & not near & - & 154 \\
\hline dry & sweet & not dry & - & 128 \\
\hline other & same & not other & contradictory & 905 \\
\hline solid & hollow & not solid & - & 159 \\
\hline parallel & oblique & not parallel & - & 163 \\
\hline alive & dead & not alive & contradictory & 209 \\
\hline alive & dead & not dead & contradictory & 1244 \\
\hline optimistic & pessimistic & not optimistic & contraries & 154 \\
\hline clear & cloudy & not clear & - & 15096 \\
\hline many & few & not many & - & 9337 \\
\hline many & few & not few & - & 322 \\
\hline
\end{tabular}

\subsection{Morphological antonyms}

Triple

Class

Frequency of negated adjective

\begin{tabular}{llllr}
\hline typical & atypical & not typical & simple negation & 668 \\
\hline confident & diffident & not confident & simple negation & 817 \\
\hline similar & dissimilar & not similar & simple negation & 314 \\
\hline similar & dissimilar & not dissimilar & double negation & 1448 \\
\hline legitimate & illegitimate & not legitimate & simple negation & 182 \\
\hline material & immaterial & not material & simple negation & 219 \\
\hline permanent & impermanent & not permanent & simple negation & 374 \\
\hline practical & impractical & not practical & simple negation & 1512 \\
\hline probable & improbable & not probable & simple negation & 139 \\
\hline
\end{tabular}




\begin{tabular}{|c|c|c|c|c|}
\hline probable & improbable & not improbable & double negation & 199 \\
\hline proper & improper & not proper & simple negation & 374 \\
\hline active & inactive & not active & simple negation & 773 \\
\hline comparable & incomparable & not comparable & simple negation & 478 \\
\hline correct & incorrect & not correct & simple negation & 1008 \\
\hline corrupt & incorrupt & not corrupt & simple negation & 133 \\
\hline credible & incredible & not credible & simple negation & 294 \\
\hline definite & indefinite & not definite & simple negation & 109 \\
\hline direct & indirect & not direct & simple negation & 397 \\
\hline efficient & inefficient & not efficient & simple negation & 185 \\
\hline flexible & inflexible & not flexible & simple negation & 199 \\
\hline offensive & inoffensive & not offensive & simple negation & 209 \\
\hline sensible & insensible & not sensible & simple negation & 246 \\
\hline substantial & insubstantial & not substantial & simple negation & 128 \\
\hline sufficient & insufficient & not sufficient & simple negation & 6102 \\
\hline valid & invalid & not valid & simple negation & 1856 \\
\hline visible & invisible & not visible & simple negation & 2238 \\
\hline responsible & irresponsible & not responsible & simple negation & 11454 \\
\hline political & nonpolitical & not political & simple negation & 490 \\
\hline traditional & nontraditional & not traditional & simple negation & 250 \\
\hline violent & nonviolent & not violent & simple negation & 144 \\
\hline able & unable & not able & simple negation & 18816 \\
\hline afraid & unafraid & not afraid & simple negation & 4345 \\
\hline ashamed & unashamed & not ashamed & simple negation & 986 \\
\hline attractive & unattractive & not attractive & simple negation & 223 \\
\hline available & unavailable & not available & simple negation & 32704 \\
\hline christian & unchristian & not christian & simple negation & 252 \\
\hline civil & uncivil & not civil & simple negation & 174 \\
\hline common & uncommon & not common & simple negation & 1858 \\
\hline common & uncommon & not uncommon & double negation & 7282 \\
\hline democratic & undemocratic & not democratic & simple negation & 127 \\
\hline desirable & undesirable & not desirable & simple negation & 481 \\
\hline due & undue & not due & simple negation & 3543 \\
\hline familiar & unfamiliar & not familiar & simple negation & 2970 \\
\hline familiar & unfamiliar & not unfamiliar & double negation & 130 \\
\hline fashionable & unfashionable & not fashionable & simple negation & 108 \\
\hline fit & unfit & not fit & simple negation & 1222 \\
\hline happy & unhappy & not happy & simple negation & 6683 \\
\hline happy & unhappy & not unhappy & double negation & 119 \\
\hline helpful & unhelpful & not helpful & simple negation & 835 \\
\hline identifiable & unidentifiable & not identifiable & simple negation & 136 \\
\hline impressive & unimpressive & not impressive & simple negation & 115 \\
\hline just & unjust & not just & simple negation & 114 \\
\hline kind & unkind & not kind & simple negation & 119 \\
\hline like & unlike & not like & simple negation & 370 \\
\hline limited & unlimited & not limited & simple negation & 7159 \\
\hline limited & unlimited & not unlimited & double negation & 117 \\
\hline loving & unloving & not loving & simple negation & 112 \\
\hline lucky & unlucky & not lucky & simple negation & 199 \\
\hline married & unmarried & not married & simple negation & 147 \\
\hline necessary & unnecessary & not necessary & simple negation & 11293 \\
\hline popular & unpopular & not popular & simple negation & 1210 \\
\hline profitable & unprofitable & not profitable & simple negation & 281 \\
\hline ready & unready & not ready & simple negation & 4060 \\
\hline realistic & unrealistic & not realistic & simple negation & 455 \\
\hline
\end{tabular}




\begin{tabular}{|c|c|c|c|c|}
\hline responsive & unresponsive & not responsive & simple negation & 148 \\
\hline satisfactory & unsatisfactory & not satisfactory & simple negation & 566 \\
\hline scientific & unscientific & not scientific & simple negation & 271 \\
\hline skilled & unskilled & not skilled & simple negation & 105 \\
\hline social & unsocial & not social & simple negation & 248 \\
\hline willing & unwilling & not willing & simple negation & 3098 \\
\hline worthy & unworthy & not worthy & simple negation & 1229 \\
\hline historical & ahistorical & not historical & simple negation & 256 \\
\hline continuous & discontinuous & not continuous & simple negation & 280 \\
\hline honest & dishonest & not honest & simple negation & 142 \\
\hline legal & illegal & not legal & simple negation & 896 \\
\hline legal & illegal & not illegal & double negation & 1001 \\
\hline logical & illogical & not logical & simple negation & 178 \\
\hline mature & immature & not mature & simple negation & 128 \\
\hline moral & immoral & not moral & simple negation & 137 \\
\hline perfect & imperfect & not perfect & simple negation & 2072 \\
\hline permissible & impermissible & not permissible & simple negation & 495 \\
\hline personal & impersonal & not personal & simple negation & 404 \\
\hline explicit & implicit & not explicit & simple negation & 271 \\
\hline possible & impossible & not possible & simple negation & 27653 \\
\hline possible & impossible & not impossible & double negation & 2668 \\
\hline precise & imprecise & not precise & simple negation & 246 \\
\hline pure & impure & not pure & simple negation & 369 \\
\hline accessible & inaccessible & not accessible & simple negation & 1795 \\
\hline accurate & inaccurate & not accurate & simple negation & 624 \\
\hline adequate & inadequate & not adequate & simple negation & 927 \\
\hline admissible & inadmissible & not admissible & simple negation & 164 \\
\hline advisable & inadvisable & not advisable & simple negation & 919 \\
\hline appropriate & inappropriate & not appropriate & simple negation & 3944 \\
\hline appropriate & inappropriate & not inappropriate & double negation & 135 \\
\hline capable & incapable & not capable & simple negation & 2320 \\
\hline compatible & incompatible & not compatible & simple negation & 2204 \\
\hline compatible & incompatible & not incompatible & double negation & 334 \\
\hline competent & incompetent & not competent & simple negation & 429 \\
\hline complete & incomplete & not complete & simple negation & 2019 \\
\hline conclusive & inconclusive & not conclusive & simple negation & 528 \\
\hline consistent & inconsistent & not consistent & simple negation & 1511 \\
\hline consistent & inconsistent & not inconsistent & double negation & 710 \\
\hline constant & inconstant & not constant & simple negation & 396 \\
\hline convenient & inconvenient & not convenient & simple negation & 371 \\
\hline decisive & indecisive & not decisive & simple negation & 119 \\
\hline dependent & independent & not dependent & simple negation & 1650 \\
\hline dependent & independent & not independent & double negation & 678 \\
\hline discriminate & indiscriminate & not discriminate & simple negation & 1669 \\
\hline distinct & indistinct & not distinct & simple negation & 191 \\
\hline divisible & indivisible & not divisible & simple negation & 176 \\
\hline effective & ineffective & not effective & simple negation & 1218 \\
\hline eligible & ineligible & not eligible & simple negation & 4253 \\
\hline exact & inexact & not exact & simple negation & 323 \\
\hline formal & informal & not formal & simple negation & 192 \\
\hline frequent & infrequent & not frequent & simple negation & 156 \\
\hline frequent & infrequent & not infrequent & double negation & 177 \\
\hline secure & insecure & not secure & simple negation & 452 \\
\hline sensitive & insensitive & not sensitive & simple negation & 435 \\
\hline significant & insignificant & not significant & simple negation & 990 \\
\hline
\end{tabular}




\begin{tabular}{|c|c|c|c|c|}
\hline significant & insignificant & not insignificant & double negation & 430 \\
\hline vulnerable & invulnerable & not vulnerable & simple negation & 185 \\
\hline rational & irrational & not rational & simple negation & 152 \\
\hline reducible & irreducible & not reducible & simple negation & 146 \\
\hline regular & irregular & not regular & simple negation & 315 \\
\hline acceptable & unacceptable & not acceptable & simple negation & 3487 \\
\hline attributable & unattributable & not attributable & simple negation & 235 \\
\hline aware & unaware & not aware & simple negation & 7791 \\
\hline aware & unaware & not unaware & double negation & 128 \\
\hline certain & uncertain & not certain & simple negation & 2893 \\
\hline characteristic & uncharacteristic & not characteristic & simple negation & 115 \\
\hline clean & unclean & not clean & simple negation & 227 \\
\hline clear & unclear & not clear & simple negation & 15096 \\
\hline comfortable & uncomfortable & not comfortable & simple negation & 755 \\
\hline conscious & unconscious & not conscious & simple negation & 297 \\
\hline conventional & unconventional & not conventional & simple negation & 131 \\
\hline critical & uncritical & not critical & simple negation & 406 \\
\hline easy & uneasy & not easy & simple negation & 9747 \\
\hline enforceable & unenforceable & not enforceable & simple negation & 170 \\
\hline enthusiastic & unenthusiastic & not enthusiastic & simple negation & 199 \\
\hline equal & unequal & not equal & simple negation & 1496 \\
\hline fair & unfair & not fair & simple negation & 1597 \\
\hline fair & unfair & not unfair & double negation & 122 \\
\hline favorable & unfavorable & not favorable & simple negation & 108 \\
\hline free & unfree & not free & simple negation & 2270 \\
\hline friendly & unfriendly & not friendly & simple negation & 109 \\
\hline healthy & unhealthy & not healthy & simple negation & 264 \\
\hline important & unimportant & not important & simple negation & 1941 \\
\hline important & unimportant & not unimportant & double negation & 111 \\
\hline intelligent & unintelligent & not intelligent & simple negation & 165 \\
\hline interested & uninterested & not interested & simple negation & 5241 \\
\hline interesting & uninteresting & not interesting & simple negation & 172 \\
\hline lawful & unlawful & not lawful & simple negation & 347 \\
\hline lawful & unlawful & not unlawful & double negation & 162 \\
\hline likely & unlikely & not likely & simple negation & 6134 \\
\hline likely & unlikely & not unlikely & double negation & 318 \\
\hline natural & unnatural & not natural & simple negation & 590 \\
\hline noticeable & unnoticeable & not noticeable & simple negation & 162 \\
\hline official & unofficial & not official & simple negation & 463 \\
\hline original & unoriginal & not original & simple negation & 518 \\
\hline pleasant & unpleasant & not pleasant & simple negation & 296 \\
\hline pleasant & unpleasant & not unpleasant & double negation & 146 \\
\hline predictable & unpredictable & not predictable & simple negation & 132 \\
\hline productive & unproductive & not productive & simple negation & 117 \\
\hline professional & unprofessional & not professional & simple negation & 307 \\
\hline real & unreal & not real & simple negation & 1434 \\
\hline reasonable & unreasonable & not reasonable & simple negation & 664 \\
\hline reasonable & unreasonable & not unreasonable & double negation & 1057 \\
\hline recoverable & unrecoverable & not recoverable & simple negation & 181 \\
\hline reliable & unreliable & not reliable & simple negation & 438 \\
\hline restricted & unrestricted & not restricted & simple negation & 294 \\
\hline sound & unsound & not sound & simple negation & 146 \\
\hline stable & unstable & not stable & simple negation & 343 \\
\hline successful & unsuccessful & not successful & simple negation & 2361 \\
\hline supportive & unsupportive & not supportive & simple negation & 205 \\
\hline
\end{tabular}




\begin{tabular}{llllr}
\hline sure & unsure & not sure & simple negation & 40753 \\
\hline sympathetic & unsympathetic & not sympathetic & simple negation & 146 \\
\hline usual & unusual & not usual & simple negation & 366 \\
\hline usual & unusual & not unusual & double negation & 4317 \\
\hline welcome & unwelcome & not welcome & simple negation & 654 \\
\hline considerable & inconsiderable & not inconsiderable & double negation & 593 \\
\hline related & unrelated & not unrelated & double negation & 173 \\
\hline fallible & infallible & not infallible & double negation & 214 \\
\hline finite & infinite & not infinite & double negation & 127 \\
\hline expected & unexpected & not unexpected & double negation & 384 \\
\hline known & unknown & not unknown & double negation & 1124
\end{tabular}

\section{References}

van Son, Chantal, Emiel van Miltenburg, and Roser Morante Vallejo. 2016. Building a dictionary of affixal negations. In Proceedings of the Workshop on Extra-Propositional Aspects of Meaning in Computational Linguistics. 